\title{
Coronary Flow Reserve Assessment of Orbital Atherectomy: An Observational Pilot Study
}

\author{
Nabil Dib"1,2, Brad J. Martinsen ${ }^{3}$ (), Bynthia M. Anose ${ }^{3}$, John McB. Hodgson ${ }^{4}$, Robert Kohler2, \\ Georges Nseir ${ }^{5}$, Nathalie R. Antonios ${ }^{6}$, Richard Shlofmitz ${ }^{7}$, Jeffrey W. Chambers ${ }^{8}$
}

${ }^{1}$ Cardiovascular Research and New Devices, Mercy Gilbert Medical Center, Gilbert, USA; ${ }^{2}$ International Society for Cardiovascular Translational Research, Phoenix, USA; ${ }^{3}$ Scientific Affairs, Cardiovascular Systems, Inc., St. Paul, USA; ${ }^{4}$ Technology Solutions Group, Moreland Hills, USA; ${ }^{5}$ Premier Cardiovascular Center, Dignity Health Chandler Hospital, Chandler, USA; ${ }^{6}$ Chicago Medical School, North Chicago, USA; ${ }^{7}$ Department of Cardiology, St. Francis Hospital, Heart Center, Roslyn, USA; ${ }^{8}$ Metropolitan Heart and Vascular Institute, Mercy Hospital, Minneapolis, USA

Correspondence to: Nabil Dib, ndib@isctr.org

Keywords: Percutaneous Coronary Intervention, Microvascular Function, Orbital Atherectomy, Calcified Lesions, Coronary Flow Reserve

Received: March 28, 2019 Accepted: April 25, $2019 \quad$ Published: April 28, 2019

Copyright $\odot 2019$ by authors and Scientific Research Publishing Inc.

This work is licensed under the Creative Commons Attribution International License (CC BY 4.0).

http://creativecommons.org/licenses/by/4.0/

\section{Open Access}

\section{ABSTRACT}

The aim of this prospective observational pilot study was to observe the impact of orbital atherectomy (OA) on the coronary microcirculation via coronary flow reserve (CFR) measurements. Fifteen subjects who had successful $O A$ and stent placement with no procedural complication were enrolled at 3 hospitals in the U.S. Baseline and hyperemic velocities were $16 \pm 5.2$ and $36 \pm 14 \mathrm{~cm} / \mathrm{sec}$, respectively. The average CFR post-procedure was within the normal range at $2.23 \pm 0.33$. The observation of normal CFR following OA may be attributed to the orbital action of the device that allows for continuous flow during treatment, minimizing a bolus embolization effect which can impact microvascular function.

\section{INTRODUCTION}

Percutaneous coronary intervention (PCI) therapies have been shown to yield successful outcomes in even the most complex interventional procedures [1]. Despite advances in equipment and technique, however, there are complex patient populations who remain challenging to treat. For example, an increased amount of calcium is associated with a higher incidence of major adverse cardiac events (MACE) when compared with non-calcified lesions [2]. Additionally, lesion calcification has been associated with vessel dissection during PCI [3], un-dilatable lesions [4], failure to deliver a stent and balloon ruptures [5].

Unlike PCI and stenting techniques in which the calcified lesion is cracked and the stenotic lesion materials are crushed into the arterial walls, atherectomy procedures reduce lesion substrate, particularly 
the calcified portions of the lesion [6]. Atherectomy vessel preparation and lesion modification, as opposed to simple balloon displacement of stenotic material, could potentially lead to reduced acute vascular trauma, lessen the stimulus for neointimal hyperplasia, and facilitate stent delivery and expansion [6-9]. Adequate plaque preparation or modification may improve both acute and long-term outcomes in subjects with complex lesions, especially heavily calcified lesions $[10,11]$. Unfortunately, reduced flow and no reflow are complications known to be associated with such approaches, specifically with rotational atherectomy (RA; Rotablator Rotational Atherectomy System; Boston Scientific; Marlborough, MA) and may indicate dysfunction of the coronary microcirculation post treatment [12].

Considering the crucial role that the coronary microcirculation plays in maintaining healthy cardiac function, it is imperative that such abnormalities be readily identified [13]. The measurement of intracoronary Doppler-derived coronary flow velocity is a useful tool to aid in elucidation of coronary flow reserve (CFR) after a PCI procedure. This approach can reveal the functional status of the coronary microcirculation, regardless of the apparent state of myocardial perfusion suggested by angiographic imaging [14].

This pilot study is the first published observations regarding the impact of Diamondback $360^{\circ}$ Coronary Orbital Atherectomy System (OA; Cardiovascular Systems, Inc.; St. Paul, MN) on coronary microvasculature function.

\section{METHODS}

\subsection{Study Design}

The Coronary Flow Reserve Following Orbital Atherectomy Study was sponsored by Cardiovascular Systems, Inc. The study was conducted according to the principles expressed in the Declaration of Helsin$\mathrm{ki}$ and was approved by each institutional review/ethics committee (ClinicalTrials.gov Identifier: NCT02339545 and Clinical Trial Registration: https://clinicaltrials.gov/ct2/show/NCT02339545). Since this was an observational single arm pilot study, no power calculations were utilized to determine sample size. The investigators decided to enroll 5 subjects from each of the three sites, totaling 15 subjects. The Diamondback Coronary Orbital Atherectomy System device (Figure 1) and mechanism of action has been described previously [6].

\subsection{Key Inclusion and Exclusion Criteria}

Key inclusion criteria required subjects to be scheduled for percutaneous coronary intervention involving stent deployment of de novo coronary lesions; subjects must have severe coronary calcification $>$ $15 \mathrm{~mm}$, vessel diameter $>2.5 \mathrm{~mm}$ and have had successful coronary stent placement with no procedural complication; subjects must have at least one unobstructed ( $\leq 40 \%$ luminal narrowing) and technically accessible coronary artery in a non-bypass grafted vessel into which a commercially available device that measures CFR could be introduced.

Key exclusion criteria included previous myocardial infarction of the target vessel; chronic renal failure or a serum creatinine level $>2.5$; previous coronary artery bypass surgery; more than 1 lesion requiring intervention; target vessel has angiographically visible or suspected thrombus; target lesion is a bifurcation. Each included target lesion was treated with coronary OA followed by stent placement.

\subsection{Observational Endpoint 1: Coronary Flow Reserve}

The orbital atherectomy and stenting procedure was considered successful if there was no residual stenosis in the index vessel $>50 \%$ diameter and there were no complications related to the index stent placement. Successful cases then underwent CFR measurement. A FloWire Doppler Guide Wire (Volcano Corporation; Rancho Cordova, CA) was advanced a minimum of $2 \mathrm{~cm}$ distal to the stent. Intracoronary nitroglycerine was administered, and after obtaining a stable signal, coronary blood flow velocity was continuously monitored. 
OAS Pump

- Mounts directly on to an iv pole

- Provides power

- Delivers fluid

- Includes saline sensor

\section{Device Features}

- Only device indicated for severe calcium

- Easy setup and use $<2$ mins

- Control of device in operating field

- Compatible with 6 Fr approach

\section{On-handle speed control}

- Low (80K) and High Speed (120K)

\section{ViperSlide ${ }^{\circledR}$ Lubricant}

- ViperSlide reduces friction during operation

- $20 \mathrm{ml}$ ViperSlide per liter of saline

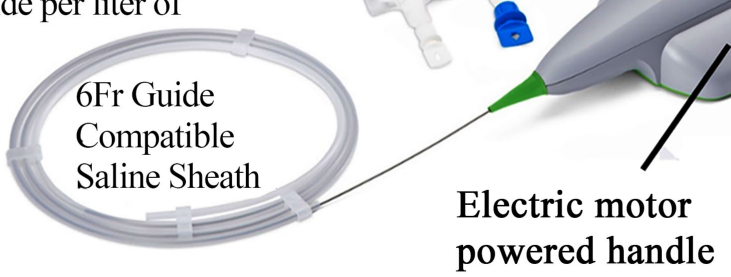

0.012 Viper Wire Advance $^{\circledR}$

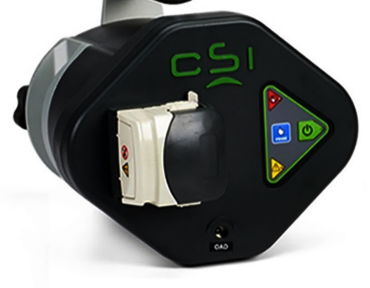

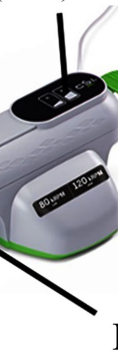

Power on/off switch

- $8 \mathrm{~cm}$ axial travel knob powered handle

Eccentrically mounted diamond-coated $1.25 \mathrm{~mm}$ classic crown

Figure 1. Diamondback 360 Coronary Orbital Atherectomy System (OAS).

Baseline and hyperemic coronary flow measurements were recorded in the index vessel. Subjects received escalating doses of intracoronary adenosine to establish their maximal coronary blood flow velocity. All velocity measurements were repeated at least twice and recorded. If the CFR values were not $\geq 2$, the operator then checked CFR in a non-stenotic vessel to allow calculation of a relative CFR.

CFR was calculated from coronary blood flow velocity measurements, which were derived from Doppler-velocity wire data recorded in real time via a dedicated console [15]. The average peak velocity $(\mathrm{APV})$ at baseline $(\mathrm{APVb})$, and peak hyperemia $(\mathrm{APVp})$ were automatically determined by integrated software.

CFR is classically measured as a ratio of two volumetric blood flow measures. Volumetric flow is the product of the flow velocity and the cross-sectional area of the vessel. Since the Doppler wire position is unchanged when measuring baseline and hyperemic velocities, and since adenosine does not induce epicardial vasodilation, the area of the vessel is assumed to be unchanged. Thus, the CFR formula utilized was

$$
\mathrm{APVp} / \mathrm{APVb}=\mathrm{CFR} \text {. }
$$

In cases where CFR was $<2.0$ and a non-stenotic vessel was also assessed, a relative CFR was determined as the ratio of CFR in the index vessel to CFR in the non-stenotic vessel. Data are expressed as mean \pm one standard deviation. CFR data were analyzed by an independent core lab (Technology Solutions Group, Moreland Hills, $\mathrm{OH}$ ).

\subsection{Observational Endpoint 2: In-Hospital Major Adverse Cardiac Events}

MACE during hospitalization was defined as cardiac death, acute myocardial infarction (Q wave or non-Q wave), and target vessel revascularization. MACE was measured at 24 hours or at discharge, whichever was earlier. 


\section{RESULTS}

\subsection{Patient and Procedure Characteristics}

Between July 2015 and May 2016, fifteen consecutive patients were enrolled at 3 hospitals in the U.S. Key baseline demographics for the subjects and lesions are shown in Table 1.

\subsection{Procedural Outcomes}

No MACE occurred in any of the 15 patients (Table 2), nor were there any episodes of slow flow, no-reflow, or hypotension observed. In addition, the average stenosis was reduced to $1.1 \% \pm 4.0 \%$ post-procedure (Table 2).

Table 1. Baseline subject and lesion demographics.

\begin{tabular}{cc}
\hline Characteristic & Total $(\mathrm{N}=15)$ \\
Age (years) & $70.6 \pm 9.3$ \\
Male & $13(86.7)$ \\
Diabetes & $5(33.3)$ \\
Hypertension & $10(66.7)$ \\
Angina & $14(93.3)$ \\
Estimated glomerular filtration rate $\left(\mathrm{mL} / \mathrm{min} / 1.73 \mathrm{~m}^{2}\right)$ & $74.6 \pm 12.1$ \\
Target lesion vessel & \\
Left anterior descending artery & $9(60.0)$ \\
Left circumflex artery & $2(13.3)$ \\
Right coronary artery & $4(26.7)$ \\
Pre-procedure minimum lumen diameter $(\mathrm{mm})$ & $0.5 \pm 0.3$ \\
Pre-procedure reference vessel diameter $(\mathrm{mm})$ & $3.3 \pm 0.4$ \\
Pre-procedure total length of calcium $(\mathrm{mm})$ & $24.6 \pm 10.3$ \\
Pre-procedure average stenosis $(\%)$ & $84.4 \pm 8.3$ \\
\hline
\end{tabular}

Values are mean \pm standard deviation or $\mathrm{n} / \mathrm{N}(\%)$.

Table 2. Post-procedural outcomes.

\begin{tabular}{cc}
\hline Outcome & Total $(\mathrm{N}=15)$ \\
\hline Post-procedure average stenosis (\%) & $1.1 \pm 4.0$ \\
In-hospital major adverse cardiac events & $0(0)$ \\
Cardiac death & $0(0)$ \\
Myocardial infarction (Q-wave or non-Q-wave) & $0(0)$ \\
Target vessel revascularization & $0(0)$ \\
Post-procedure APVb (cm/s) & Total $(\mathrm{N}=8)$ \\
Post-procedure APVp (cm/s) & $16.0 \pm 5.2$ \\
Post-procedure CFR (ratio) & $36.0 \pm 14.0$ \\
\hline
\end{tabular}

Values are mean \pm standard deviation or $\mathrm{n} / \mathrm{N}(\%)$. 
$\mathrm{APVb}$ and $\mathrm{APVp}$ readings were measured after each index procedure $(\mathrm{OA}+$ stent $)$ in the 15 subjects and valid readings were used to calculate CFR. Only 8 of the 15 subjects resulted in valid velocity and CFR measurements related to the OA + stent treatment; therefore, 7 subjects were excluded from the final CFR analysis. Briefly, four of the subjects were excluded by the core lab due to poor quality APVb and/or APVp readings. An additional two subjects were excluded per the protocol-the relative ratio of index/normal vessel CFR yielded a value of approximately 1.0 (i.e. these patients presumably had intrinsic microvascular dysfunction, unrelated to the OA + stent intervention). Lastly, one additional subject was excluded due to missing data (malfunction of the measuring device), as reported by the operator.

The overall mean post-procedural APVb, APVp, and CFR (ratio) were $16 \pm 5.2 \mathrm{~cm} / \mathrm{s}, 36 \pm 14 \mathrm{~cm} / \mathrm{s}$, and $2.23 \pm 0.33$, respectively, for the final 8 subjects included in CFR analysis (Table 2).

\section{DISCUSSION}

Recent evidence has demonstrated that functional abnormalities, including those caused by PCI [16], can result in abnormal coronary microvascular function. Diminishment of CFR may have significant acute and chronic physiological consequences. For example, increases in creatine kinase (CK > 1x ULN) and cardiac troponin $\mathrm{T}$ markers were shown to occur at a rate $6 \mathrm{x}$ and $9 \mathrm{x}$ higher, respectively, in patients who experienced persistent CFR depression after PCI than those who did not. This strongly indicates the presence of myocardial injury and suggests procedural embolization of atherothrombotic debris as a potential underlying mechanism [17].

Unlike previous studies showing depressed CFR post RA treatment [12], the results shown here indicate a mean CFR reading in the normal range (>2.0) post OA treatment, as well as a zero incidence of slow flow/no-reflow complications. These results are consistent with the ORBIT II study (Evaluate the Safety and Efficacy of OAS in Treating Severely Calcified Coronary Lesions) that showed very low rates of persistent slow-flow/no-reflow after OA, as well as low rates of Q-wave and non Q-wave myocardial infarction rates [10]. These results may be due to the orbital action of the device that allows for continuous flow during treatment, minimizing a bolus embolization effect which can impact microvascular function [6].

Lastly, recent research comparing OA versus RA utilizing another measure of coronary microvascular function called index of myocardial resistance (IMR) indicated significantly lower IMR in the OA group compared to the RA group, suggesting preserved microvascular function post OA [18]. The CFR observational pilot study reported herein and the recent IMR results has generated the hypothesis that OA results in better microvasculature function post treatment than RA. To test this hypothesis, the prospective ORACLE (Comparison of Orbital Versus Rotational Atherectomy Effects on Coronary Microcirculation in PCI) randomized control trial $(\mathrm{n}=40)$ was designed and is currently enrolling subjects. The ORACLE trial will use IMR, post-procedural cardiac enzymes, and cardiac MRI to assess microvascular abnormalities after atherectomy (ClinicalTrials.gov Identifier: NCT03021577).

This was a small, non-randomized, pilot study designed only for observational and hypothesis generating evidence. In addition, neither comparison with RA nor PCI without atherectomy was performed. All procedures were performed by four operators at three institutions, and, thus, results may not be generalizable to all medical centers and operators considering a difference in training and expertise. Randomized trials are needed to clearly determine the impact of OA on coronary microvascular function compared to other PCI techniques.

\section{CONCLUSION}

The observation of normal CFR post OA may be attributed to the orbital action of the device that allows for continuous flow during treatment, minimizing a bolus embolization effect which can impact microvascular function.

\section{ACKNOWLEDGEMENTS}

The authors thank Ann Behrens, BS, of Cardiovascular Systems, Inc. (CSI) for editing and critical re- 
view of this manuscript.

\section{CONFLICTS OF INTEREST}

Drs. Nabil Dib, Richard Shlofmitz, and Jeff Chambers receive consulting fees from CSI; Drs. Brad Martinsen and Bynthia Anose are employed by and own stock in CSI; Dr. John McB. Hodgson, Robert Kohler, Dr. Georges Nseir, and Dr. Nathalie Antonios have no relevant relationships to disclose.

\section{REFERENCES}

1. Bass, T.A. (2017) Percutaneous Coronary Interventions for Chronic Total Occlusions: As the Technology Expands, Our Responsibilities Increase. Circulation, 135, 1385-1387.

https://doi.org/10.1161/CIRCULATIONAHA.116.022151

2. Généreux, P., Madhavan, M.V., Mintz, G.S., Maehara, A., Palmerini, T., Lasalle, L., et al. (2014) Ischemic Outcomes after Coronary Intervention of Calcified Vessels in Acute Coronary Syndromes: Pooled Analysis from the Horizons-AMI (Harmonizing Outcomes with Revascularization and Stents in Acute Myocardial Infarction) and Acuity (Acute Catheterization and Urgent Intervention Triage Strategy) Trials. Journal of the American College of Cardiology, 63, 1845-1854. https://doi.org/10.1016/j.jacc.2014.01.034

3. Benezet, J., Diaz de la Llera, L.S., Cubero, J.M., Villa, M., Fernandez-Quero, M. and Sanchez-Gonzalez, A. (2011) Drug-Eluting Stents Following Rotational Atherectomy for Heavily Calcified Coronary Lesions: Long-Term Clinical Outcomes. Journal of Invasive Cardiology, 23, 28-32.

4. Schlüter, M., Cosgrave, J., Tübler, T., Melzi, G., Colombo, A. and Schofer, J. (2007) Rotational Atherectomy to Enable Sorolimus-Eluting Stent Implantation in Calcified, Nondilatable De Novo Coronary Artery Lesions. Vascular Disease Management, 4, 63-69.

5. Moussa, I., Ellis, S.G., Jones, M., Kereiakes, D.J., McMartin, D., Rutherford, B., et al. (2005) Impact of Coronary Culprit Lesion Calcium in Patients Undergoing Paclitaxel-Eluting Stent Implantation (A Taxus-IV Sub Study). American Journal of Cardiology, 96, 1242-1247. https://doi.org/10.1016/j.amjcard.2005.06.064

6. Shlofmitz, E., Martinsen, B.J., Lee, M., Rao, S.V., Généreux, P., Higgins, J., et al. (2017) Orbital Atherectomy for the Treatment of Severely Calcified Coronary Lesions: Evidence, Technique, and Best Practices. Expert Review of Medical Devices, 14, 867-879. https://doi.org/10.1080/17434440.2017.1384695

7. Chambers, J.W., Behrens, A.N. and Martinsen, B.J. (2016) Atherectomy Devices for the Treatment of Calcified Coronary Lesions. Interventional Cardiology Clinics, 5, 143-151. https://doi.org/10.1016/j.iccl.2015.12.003

8. Chambers, J.W. and Martinsen, B.J. (2016) Orbital Atherectomy in the Coronary Arteries. In: Shammas, N.W., Ed., Textbook of Atherectomy, HMP Communications, 217-240.

9. Lee, M.S. and Shah, N. (2016) The Impact and Pathophysiologic Consequences of Coronary Artery Calcium Deposition in Percutaneous Coronary Interventions. Journal of Invasive Cardiology, 28, 160-167.

10. Chambers, J.W., Feldman, R.L., Himmelstein, S.I., Bhatheja, R., Villa, A.E., Strickman, N.E., et al. (2014) Pivotal Trial to Evaluate the Safety and Efficacy of the Orbital Atherectomy System in Treating De Novo, Severely Calcified Coronary Lesions (ORBIT II). JACC: Cardiovascular Interventions, 7, 510-518. https://doi.org/10.1016/j.jcin.2014.01.158

11. Hoffmann, R., Mintz, G.S., Kent, K.M., Pichard, A.D., Satler, L.F., Popma, J.J., et al. (1998) Comparative Early and Nine-Month Results of Rotational Atherectomy, Stents, and the Combination of Both for Calcified Lesions in Large Coronary Arteries. American Journal of Cardiology, 81, 552-557. https://doi.org/10.1016/S0002-9149(97)00983-1

12. Tanaka, T., Shimizu, Y., Ishihara, A., Yano, K., Sada, T. and Kira, Y. (2005) Effect of Rotational Atherectomy on the Coronary Microcirculation in Patients with Angina Pectoris. Journal of Cardiology, 46, 43-51. 
13. Flammer, A.J., Anderson, T., Celermajer, D.S., Creager, M.A., Deanfield, J., Ganz, P., et al. (2012) The Assessment of Endothelial Function: From Research into Clinical Practice. Circulation, 126, 753-767. https://doi.org/10.1161/CIRCULATIONAHA.112.093245

14. Pries, A.R., Habazettl, H., Ambrosio, G., Hansen, P.R., Kaski, J.C., Schächinger, V., et al. (2008) A Review of Methods for Assessment of Coronary Microvascular Disease in Both Clinical and Experimental Settings. Cardiovascular Research, 80, 165-174. https://doi.org/10.1093/cvr/cvn136

15. Díez-Delhoyo, F., Gutiérrez-Ibañes, E., Loughlin, G., Sanz-Ruiz, R., Vázquez-Álvarez, M.E., Sarnago-Cebada, F., et al. (2015) Coronary Physiology Assessment in the Catheterization Laboratory. World Journal of Cardiology, 7, 525-538. https://doi.org/10.4330/wjc.v7.i9.525

16. Herrmann, J., Kaski, J.C. and Lerman, A. (2012) Coronary Microvascular Dysfunction in the Clinical Setting: From Mystery to Reality. European Heart Journal, 33, 2771-2782. https://doi.org/10.1093/eurheartj/ehs246

17. Herrmann, J., Haude, M., Lerman, A., Schulz, R., Volbracht, L., Ge, J., et al. (2001) Abnormal Coronary Flow Velocity Reserve After Coronary Intervention is Associated with Cardiac Marker Elevation. Circulation, 103, 2339-2345. https://doi.org/10.1161/01.CIR.103.19.2339

18. Galougahi, K.K., Bhatti, N., Shlofmitz, R., Généreux, P., Moses, J., Kirtane, A., et al. (2016) TCT-236 Effects of Orbital versus Rotational Atherectomy Facilitated PCI on the Coronary Microcirculation. Journal of the American College of Cardiology, 68, B96. https://doi.org/10.1016/j.jacc.2016.09.247 